\title{
The use of LC-MS to identify differentially expressed proteins in docetaxel-resistant prostate cancer cell lines
}

\author{
Kathleen O'Connell ${ }^{1}$, Maria Prencipe ${ }^{2}$, Amanda O'Neill ${ }^{2}$, Claire Corcoran ${ }^{3}$, Sweta Rani ${ }^{3}$, \\ Michael Henry ${ }^{1}$, Paul Dowling ${ }^{1}$, Paula Meleady ${ }^{1}$, Lorraine O'Driscoll ${ }^{3}$, William Watson ${ }^{2}$ \\ and Robert $O^{\prime}$ Connor ${ }^{1,4}$ \\ ${ }^{1} \mathrm{MTCl}$ National Institute for Cellular Biotechnology, DCU, Glasnevin, Dublin, Ireland \\ ${ }^{2} \mathrm{MTCl}$ Conway Institute of Biomolecular \& Biomedical Research, UCD, Belfield, Dublin, Ireland \\ ${ }^{3} \mathrm{MTCl}$ School of Pharmacy and Pharmaceutical Sciences, Trinity College Dublin, Dublin, Ireland \\ ${ }^{4}$ School of Nursing, DCU, Glasnevin, Dublin, Ireland
}

\begin{abstract}
Docetaxel is a taxane-derived chemotherapy drug that has been approved for treatment of prostate cancer. While docetaxel is frequently used as a treatment for hormone-refractory prostate cancer, a subset of patients either do not respond to this treatment or those that do respond eventually become resistant to the drug over time. Resistance to docetaxel is complex and multi-factoral and further understanding of the cellular biochemistry underlying resistance is vital to improve treatment efficacy. To identify proteins altered in the resistant phenotype, three parental cell lines DU145, 22RV1 and PC-3, as well as their docetaxel resistant sub-lines, were subjected to quantitative label-free LC-MS proteomic profiling. A total of 189 significant $(p<0.05)$ protein abundance changes were identified in the DU145 resistant sub-lines, 254 in the 22RV1 sub-lines, and 51 and 72 in the 8 and $12 \mathrm{nM}$ resistant PC-3 sub-lines, respectively. From these, 29 proteins demonstrated a significant $(p<0.05)$ fold change across two or more resistant variants. These included proteins indicative of an epithelial-to-mesenchemyl transition as well as altered heat shock response elements.
\end{abstract}

Received: September 16, 2011

Revised: February 13, 2012

Accepted: March 5, 2012

\section{Keywords:}

Cancer / Cell biology / Docetaxel / Prostate / Resistant

\section{Introduction}

Prostate cancer is a leading cause of cancer-related mortality in males, with approximately 33720 deaths occurring in the United States in 2011 [1,2]. This malignancy primarily affects older men with the median diagnosis age being 68 years [3].

Initial prostate tumour growth is generally androgen dependent and, where indicated, therapy consists of surgery,

Correspondence: Dr. Kathleen O'Connell, Molecular Therapeutics Cancer Ireland, National Institute for Cellular Biotechnology, DCU, Glasnevin, Dublin 9, Ireland

E-mail: Kathleen.OConnell@dcu.ie

Fax: +353 (01) 7005484

Abbreviations: AR, androgen receptor; CRPC, castrationrefractory prostate cancer; EMT, epithelial-to-mesenchymal transition; PSA, prostate-specific antigen radiation and androgen ablation. Androgen ablation therapy typically results in temporary tumour regression as a result of apoptosis induction due to the loss of steroid, however, as the cancer progresses, it often switches to androgenindependent growth, within 2-3 years, thereby becoming refractory to further hormonal ablation [4]. This is known as castration-refractory prostate cancer (CRPC). Despite the lack of response to androgen ablation, the androgen receptor (AR) continues to be expressed after progression to CRPC [5]. Therefore, mechanisms other than loss of AR expression are thought to drive CRPC.

Docetaxel, a taxane chemotherapeutic, is approved for use in the treatment of advanced prostate cancer [6-8]. It induces apoptosis by binding to tubulin and stabilising microtubules and was the first drug to show survival benefits in CRPC. It is currently the most effective drug used in the treatment

Colour Online: See the article online to view Figs. 1 and 2 in colour. 
of CRPC $[6,9]$, showing modest survival benefits, with an increase in median survival time of 2 months, compared to other treatment regimens [6, 7]. However, docetaxel can display variable pharmacokinetics in patients with up to ten-fold differences in drug clearance; reduced clearance of the drug is associated with serious side effects such as neutropenia $[10,11]$. Lower severity side effects including fatigue, nausea, vomiting and alopecia are also associated with docetaxel treatment of CRPC [6].

CRPC patients treated with docetaxel eventually develop docetaxel resistance. Although some patients have the option of further docetaxel treatment after a treatment-free interval, no standard therapy for docetaxel-resistant CRPC exists [12]. Docetaxel resistance has been attributed to a number of mechanisms, including low initial effectiveness in prostate cancer cells [13], alterations in tubulin isotype expression [14], and increased AKT signalling due to loss of PTEN [15]. Elevated levels of proteins such as Bcl-2 [16], other anti-apoptotic proteins [17], STAT1 [18], clusterin [19] and HSPs [20] also contribute to resistance by decreasing the apoptotic response of cells.

Biomarkers of disease and drug resistance are key tools in the predicting progression or drug resistance in diseases such as prostate cancer. Proteomics has been applied to the discovery of mechanisms of drug resistance in cell line models and clinical samples [21] and has a critical role to play in furthering our understanding of all aspects of prostate cancer [22]. One of the best known markers of prostate cancer is the prostate-specific antigen (PSA), which is routinely measured to aid in the detection of the disease. However, elevated PSA levels are not specific to prostate cancer and this can result in an initial false diagnosis [23]. This highlights the importance of the development and use of more than one marker for diagnosis, evaluation of disease progression or drug resistance. In the case of prostate cancer, further research is needed to identify signatures of disease progression and drug resistance. Advances in technology now allows global quantitative analysis of drug resistance through genomics, proteomics and metabolomics (amongst others). This development has led to the routine use of MS as a quantitative tool in proteomics [24]. MS-based quantitative proteomics has been applied to prostate cancer in identification of new diagnostic markers [25,26], disease progression [27] and mechanisms of drug resistance [28]. To date, proteomic that which focused on docetaxel resistance in CRPC have analysed single docetaxel resistant sub-lines [28]. The aim of this study was to survey proteins in multiple prostate cancer cell lines to reveal common differences in protein expression relating to docetaxel resistance, thereby hoping to identify robust indicators of resistance that might also provide new mechanistic insight. We used a label-free LC-MS approach to compare two androgen-independent (PC-3, DU-145) cell lines and one androgen-sensitive (22RV1) cell line to their docetaxel resistant sub-lines (PC-3 D8, PC-3 D12, DU145-RD and 22RV1-RD). Resistance in all three cell lines was associated with alterations in microtubule-associated cytoskeletal proteins, suggestive of epithelial-to-mesenchymal transition (EMT), and changes to HSP levels.

\section{Materials and methods}

\subsection{Materials}

Formic acid purchased from Fluka (Buchs, Switzerland). HPLC grade acetone, methanol, ACN, water, Tris(2carboxyethyl) phosphine (TCEP), Tris, iodoacetamide, NP40 , sodium chloride, sodium deoxycholate, SDS and PBS (tablets) were obtained from Sigma-Aldrich (St. Louis, MO, USA). Sequencing grade trypsin was from Promega (Madison, WI, USA). Protease inhibitor cocktail tablets were from Roche (Basel, Switzerland). Pre-cast minigels for 1DE were purchased from Lonza (Basel, Switzerland).

Antibodies for HSP70 (ab47455), and the AR (ab9474) were from Abcam (Cambridge, UK). GRP78 antibody (610978) was from BD Transduction Laboratories (San Diego, CA, USA). Vimentin (V4630) and Vinculin (V4139) were from Sigma-Aldrich. K1C19 antibody (MAB3238) was from Chemicon/Millipore (Billerica, MA, USA). K1C18 antibody (sc-6259) was from Santa Cruz Biotechnology (Santa Cruz, CA, USA).

\subsection{Cell culture}

Human prostate cancer cell lines PC-3, DU-145 and 22RV1 were purchased from the American Type Culture Collection (ATCC) and maintained in RPMI-1640 medium supplemented with $10 \% \mathrm{FBS}, 50 \mathrm{U} / \mathrm{mL}$ penicillin $/ 50 \mu \mathrm{g} / \mathrm{mL}$ streptomycin and $2 \mathrm{mM}$ L-glutamine (Invitrogen, Carlsbad, CA, USA). PC-3, DU145 and 22RV1 resistant sub-lines were generated by treating with Docetaxel (Sigma, St. Louis, MO, USA) as previously described [29]. A subset of cells were aged alongside the resistant cells as an appropriate control (PC-3 Ag, DU145 Ag and 22RV1 Ag). In all subsequent experiments, the resistant PC-3 cells are referred to as PC-3 D8 and PC-3 D12 (reflecting the final treatment doses), while the DU145 and 22RV1 resistant sub-lines are referred to as DU145 RD and 22RV1 RD, respectively. The PC-3 Ag cells had an $\mathrm{IC}_{50}$ of $10 \mathrm{nM}$, the PC-3 D8 $\mathrm{IC}_{50}=20 \mathrm{nM}$ and the PC-3 D12 $\mathrm{IC}_{50}=100 \mathrm{nM}$ following treatment with Docetaxel [29]. The DU145Ag cells had an $\mathrm{IC}_{50}$ of $1.7 \mathrm{nM}$, the DU145 RD IC $50=$ $183.3 \mathrm{nM}, 22 \mathrm{RV} 1 \mathrm{Ag} \mathrm{IC} \mathrm{I}_{50}=4 \mathrm{nM}$ and 22Rv1 RD IC $50=277$ $\mathrm{nM}$. Biological replicates, for each cell line, were within 10 passages of each other, with age-matched parental cells harvested at the same passage as matching resistant derivative cells. Cells were passaged for a minimum of 2 weeks in the absence of docetaxel before any experiments were carried out.

\subsection{Protein extraction}

Due to the inherent variability of protein fractionation, it was decided to analyse whole cell lysates to minimise differences 
between the multiple cell lines analysed. Cells were harvested for LC-MS analysis, following five washes in PBS by scraping into cold PBS. Approximately $3 \times 10^{6}$ cells were spun at 1000 $\times \mathrm{g}$, and the cell pellet snap frozen in liquid nitrogen.

The cell pellet was resuspended in lysis buffer $(50 \mathrm{mM}$ Tris, $\mathrm{pH} 8,150 \mathrm{mM} \mathrm{NaCl}, 2 \% \mathrm{NP}-40,0.5 \%$ sodium deoxycholate and $0.1 \%$ SDS). Lysis of cells was also aided by three freeze-thaw cycles in liquid nitrogen. Protein lysates were clarified by centrifugation $\left(16000 \times \mathrm{g}, 40 \mathrm{~min}, 4^{\circ} \mathrm{C}\right)$. Protein concentration was determined by BCA protein assay kit (Pierce, Rockford, IL, USA).

\subsection{Protein digestion}

Samples were digested according to the method of Duan et al. [30]. Protein samples were reduced with $4 \mathrm{mM}$ TCEP at $95^{\circ} \mathrm{C}$ for $5 \mathrm{~min}$ and alkylated with $20 \mathrm{mM}$ iodoacetamide at $37^{\circ} \mathrm{C}$ for $30 \mathrm{~min}$. A total of $100 \mu \mathrm{g}$ of total cell lysate was precipitated in $100 \%$ percent ice-cold acetone, added in two aliquots, the first was 1:1 (v/v) protein solution:acetone and the second 1:8 initial protein solution:acetone. This was incubated overnight at $-20^{\circ} \mathrm{C}$ and then centrifuged at $12000 \times \mathrm{g}$ for $20 \mathrm{~min}$ at $4^{\circ} \mathrm{C}$. A further $100 \mu \mathrm{L}$ of acetone was used to wash the pellet and it was again centrifuged at $12000 \times \mathrm{g}$ for $20 \mathrm{~min}$ at $4^{\circ} \mathrm{C}$. The pellet was allowed to air-dry for $3 \mathrm{~min}$.

Trypsin (in $50 \mathrm{mM}$ Tris buffer, $\mathrm{pH}$ 8.5) was added at an enzyme/substrate ratio of 1:50 (w/w). After the sample was briefly $(10 \mathrm{~s})$ sonicated in a water bath sonicator, and the solution was incubated at $37^{\circ} \mathrm{C}$ and $120 \mathrm{rpm}$ for $5 \mathrm{~h}$ in a New Brunswick shaking incubator (Edison, NJ, USA). A second aliquot of trypsin was added at an enzyme/substrate ratio of $1: 50(\mathrm{w} / \mathrm{w})$ and the mixture incubated at $37^{\circ} \mathrm{C}$ overnight. This was spun at $16000 \times \mathrm{g}$ for $15 \mathrm{~min}$ and $75 \mu \mathrm{L}$ of the supernatant dried down in a vacuum centrifuge.

\subsection{MS}

Digested samples were resuspended in 0.1\% TFA (Fluka) in $2 \%$ ACN and analysed by nanoLC-MS/MS using an Ultimate 3000 system (Dionex) coupled to a nanospray LTQ Orbitrap mass spectrometer (Thermo Fisher Scientific, Rockford, IL, USA), using a linear ACN gradient from $0 \%$ to $65 \%$ ACN (Sigma) over 180 min. Buffers used for nano LC separation contained $0.1 \%$ formic acid as the ion pairing reagent. The flow rate was $300 \mathrm{~nL} / \mathrm{min}$. The LTQ Orbitrap was operated in data-dependent acquisition mode with Xcalibur software. Survey scan MS data were acquired in the Orbitrap on the $300-2000 \mathrm{~m} / z$ mass range with the resolution set to a value of 60000 at $400 \mathrm{~m} / z$. The five most intense ions per survey scan were selected for MS/MS fragmentation and the resulting fragments were analysed in the linear trap. Collision energy was set to $35 \%$. Dynamic exclusion was employed within 60 s. Full scan mass spectra were recorded in profile mode and tandem mass spectra in centroid mode. Biological replicates $(n=4)$ were analysed for each sample type.

\subsection{Progenesis analysis}

RAW files were analysed in Progenesis LC-MS software. This software extracts quantitative information from MS1 data by aligning each LC-MS run to a reference file. Results were filtered based on statistical analysis. Any peptides with an ANOVA score of $p>0.05$ were eliminated.

The MS2 data for the remaining peptides was exported and the resulting MGF file used to search the Swissprot database (Release 2011_05) on the MASCOT server (www.matrixscience.com) for protein identifications. The Mascot parameters were (1) species, Homo sapiens, (2) allowed number of missed cleavages, 1, (3) fixed modification, carboxylmethyl, (4) variable modifications, methionine oxidation (5) peptide tolerance, $\pm 20 \mathrm{ppm}$, (6) MS/MS tolerance, $\pm 0.6 \mathrm{Da}$ and (7) peptide charge, $+2,+3$ and +4 . Peptides were also searched against a decoy database to determine the false discovery rate (FDR).

The total number of unique peptides exported were 2143 (PC-3 versus PC-3 D8), 1481 (PC-3 versus PC-3 D12), 20030 (DU145 Ag versus DU145 RD) and 30425 (22RV1 Ag versus 22RV1 RD). Based on these, a total of 173 (PC-3 D8, FDR = 1.98\%), 279 (PC-3 D12, FDR = 2.54\%), 523 (DU145 RD, FDR $=3.82 \%)$ and $777(22 \mathrm{RV} 1 \mathrm{RD}, \mathrm{FDR}=2.24 \%)$ proteins were identified. This was then further narrowed down based on a mascot peptide scores.

Peptide identifications were imported into the Progenesis software and assigned to the matching features. Peptide identifications with a Mascot score less than 40 were eliminated and peptides were assigned to a protein. Protein scores were based on ANOVA values with a cut off of $p<0.05$. Proteins with quantitative information from two or more peptides were considered as significant. Peptide conflicts occur when a peptide is identified as present in more than one protein. These were resolved by assigning the peptide to the protein with the greater number of hits, a greater Mascot score or a lower mass error; when conflicts could not be clearly resolved, the peptide was excluded from the analysis.

\subsection{Pathway analysis}

A shortlist of proteins was compiled from proteins that showed a change in abundance with a similar trend in two or more docetaxel-resistant sub-lines. Protein function, regulation and interaction were investigated by analysing shortlisted proteins in Pathway Studio 8.0 [31]. This software was used to build protein networks based on in silico literature mining. When features of interest were identified the literature for that node was assessed. 


\subsection{Western blotting}

Protein samples were reduced in Laemmli buffer supplemented by $350 \mathrm{mM}$ DTT for $10 \mathrm{~min}$ at $95^{\circ} \mathrm{C}$, prior to separation of $2.5-30 \mu \mathrm{g}$ of protein on a $7.5 \%$ precast minigel. Proteins were transferred to a nitrocellulose membrane over 6 min on the dry iBlot transfer system (Invitrogen). The membrane was blocked with blocking solution (5\% dry milk in PBS) for $1 \mathrm{~h}$ at room temperature before incubation with the primary antibody in $2.5 \%$ milk (in PBS) solution at $4^{\circ} \mathrm{C}$ overnight. After two 10-min washes with blocking solution, the membrane was incubated with HRP secondary antibody for $1 \mathrm{~h}$. A further two washes in blocking solution was followed by two washes in PBS. Subsequently, the blot was visualised by incubating the blot for $5 \mathrm{~min}$ with ECL
Plus Western Blotting Detection substrate (GE Healthcare, Buckinghamshire, UK) and fluorescent emission captured by scanning blots at $457 \mathrm{~nm}$ excitation, $520 \mathrm{~nm}$ emission (PMT 450) on a Typhoon 9400 fluorescent scanner. Densitometry was carried out by assessing band volume in ImageQuant 5.2 software (Molecular Dynamics, GE Healthcare). The protein of interest was then normalised to the housekeeping protein $\beta$-actin. Significance of abundance changes was assessed by student $t$-test with a significance value cut off of $p<0.05$. All blots were assessed in three biological replicates.

Additionally, 1D gels, on which $30 \mu \mathrm{g}$ of protein was resolved, were stained with colloidal coomassie stain (Sigma) overnight and destained in $10 \%(\mathrm{v} / \mathrm{v})$ methanol to assess protein loading of lysates from each cell line.

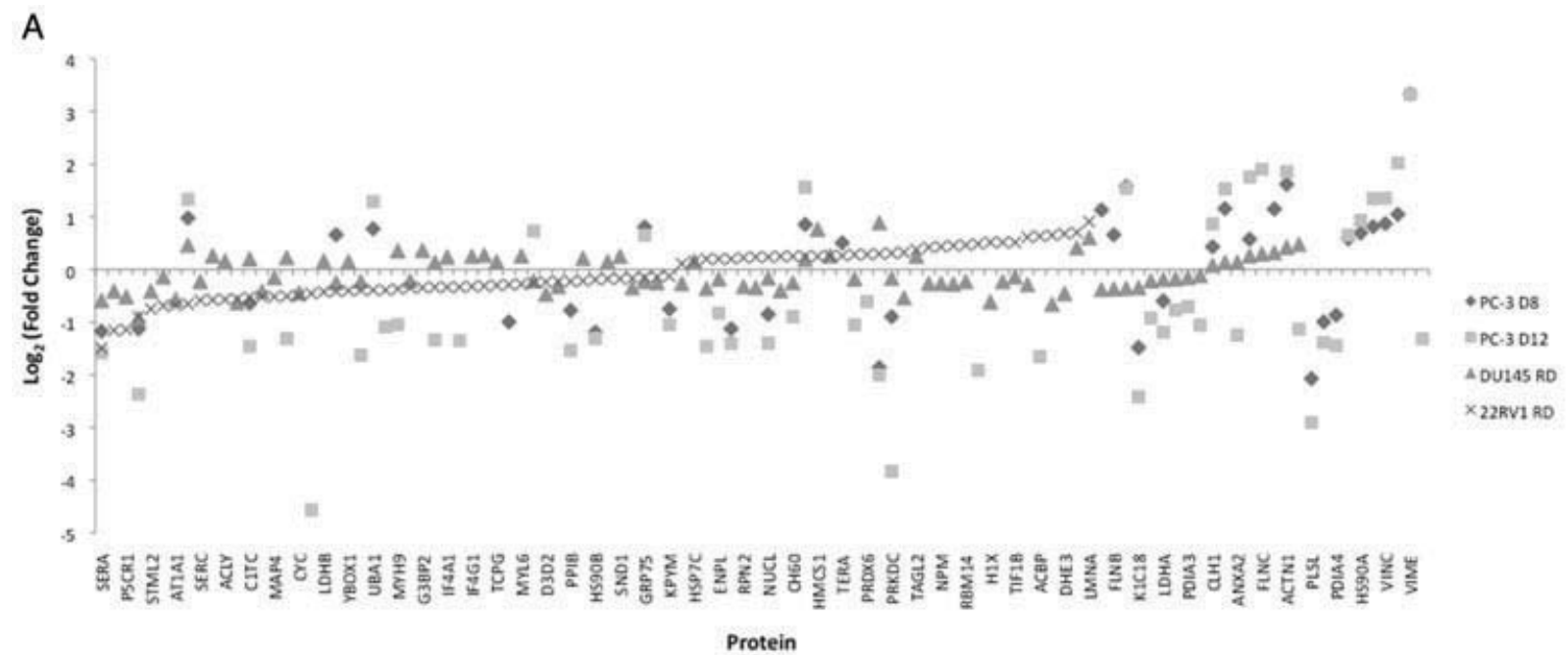

B

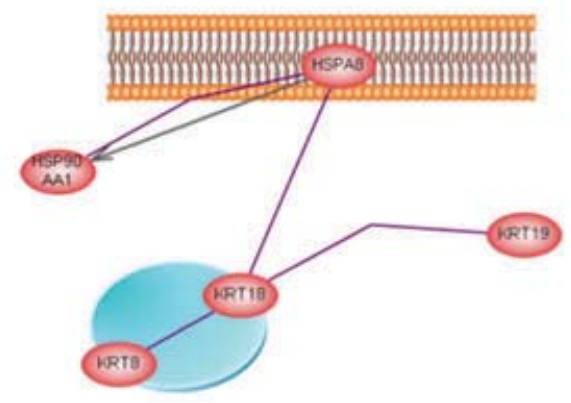

Figure 1. Global analysis of protein abundance changes. (A) Scatter plot of protein abundance changes, as determined by label-free LC-MS, in PC-3 docetaxel resistant variants (D8 and D12), DU145 and 22RV1 docetaxel resistant variants (RD). Only changes detected in more than one docetaxel resistant cell line were plotted. Fold changes were calculated on the $\log _{2}$ scale (y-axis). Proteins were arranged in order of ascending $\log _{2}$ (fold change) in the 22RV1 cell line and then the DU145 RD cell line ( $x$-axis), which represented the greatest number of changes present. (B) Graphical summary of interactions between short-listed proteins (A) as determined by literature mining (Pathway Studio 8.0). Proteins are represented by red circles. Protein cellular location is represented as membrane, cytoplasmic (white area) or nuclear (turquoise). Grey lines represent regulation of protein activity by direct interaction while purple lines represent protein binding. HSP90AA1 = HSP90A, KRT19= K1C19, KRT18 = K1C18, KRT8 = K2C8, HSPA8 = HSP71 


\section{Results}

\subsection{Proteomic profiling of docetaxel-resistant cell lines}

A total of 189 significant $(p<0.05)$ protein abundance changes were identified in the DU145 cell line, 254 in the 22RV1 cell line, and 51 and 72 in the 8 and $12 \mathrm{nM}$ resistant PC-3 variants, respectively, when compared to their sensitive parental lines. A full list of protein abundance changes detected for each cell line, as well as MS data is available in Supporting Information Tables S1-S4. Global analysis of these changes showed that a large number of proteins had altered abundance in more than one cell line, see Fig. 1(A). In the case of the two PC-3 docetaxel resistant variants (PC-3 D8 and PC-3 D12), the magnitude of the $\log _{2}$ (fold change) is related to the level of docetaxel resistance, as the more sensitive PC-3 D8 cells consistently demonstrated smaller fold changes.

\subsection{Common changes}

A short list consisting of 31 proteins present in less than two cell lines, with concurrent changes, was compiled and analysed by Pathway Studio 8.0; five proteins had interactions with each other, with several regulating the activity of others by direct interactions (grey lines) while others showed evidence of protein binding (purple lines; Fig. 1 B). As expected, interactions were shown between HSP90 and HSP71.

Seventeen proteins were identified with altered abundance levels in all PC-3, DU145 and 22RV1 docetaxel resistant sub-lines (Listed in Table 1). Of these proteins, four displayed concurrent changes in protein abundance in docetaxel resistant cells. HSP71 a $70 \mathrm{kDa}$ HSP (increased abundance), a cytokeratin (K2C8, decreased abundance), D-3-phosphoglycerate dehydrogenase (SERA, decreased abundance) and valyl-tRNA synthetase (SYVC, decreased abundance).

Table 1. Eighteen proteins with a change in abundance in either PC-3 docetaxel resistant sub-lines and both DU145 RD and $22 \mathrm{RV} 1 \mathrm{RD}$ sub-lines.

\begin{tabular}{|c|c|c|c|c|c|c|c|c|c|}
\hline \multicolumn{2}{|c|}{ Uniprot entry Protein } & \multicolumn{2}{|l|}{ PC-3 D8 } & \multicolumn{2}{|l|}{ PC-3 D12 } & \multicolumn{2}{|l|}{ DU145 RD } & \multicolumn{2}{|l|}{ 22RV1 RD } \\
\hline & & $\begin{array}{l}\log _{2} \\
\text { (fold change) }\end{array}$ & $\begin{array}{l}\text { Unique } \\
\text { peptides }\end{array}$ & $\begin{array}{l}\log _{2} \\
\text { (fold change) }\end{array}$ & $\begin{array}{l}\text { Unique } \\
\text { peptides }\end{array}$ & $\begin{array}{l}\log _{2} \\
\text { (fold change) }\end{array}$ & $\begin{array}{l}\text { Unique } \\
\text { peptides }\end{array}$ & $\begin{array}{l}\log _{2} \\
\text { (fold change) }\end{array}$ & $\begin{array}{l}\text { Unique } \\
\text { peptides }\end{array}$ \\
\hline ACTN4 & Alpha-actinin-4 & 0.98 & 7 & 1.33 & 3 & 0.46 & 21 & -0.65 & 9 \\
\hline ALDOA & $\begin{array}{l}\text { Fructose- } \\
\text { bisphosphate } \\
\text { aldolase A }\end{array}$ & 0.66 & 2 & & & -0.25 & 12 & -0.41 & 3 \\
\hline C1TC & $\begin{array}{l}\text { C-1-Tetrahydrofolate } \\
\text { synthase, } \\
\text { cytoplasmic }\end{array}$ & -0.64 & 2 & -1.45 & 8 & 0.20 & 10 & -0.53 & 3 \\
\hline EF1D & $\begin{array}{l}\text { Elongation factor } \\
\text { 1-delta }\end{array}$ & & & -1.31 & 4 & 0.23 & 9 & -0.51 & 3 \\
\hline GRP75 & $\begin{array}{l}\text { Stress-70 protein, } \\
\text { mitochondrial }\end{array}$ & 0.81 & 2 & 0.81 & 3 & -0.23 & 20 & -0.15 & 9 \\
\hline HSP71 & $\begin{array}{c}\text { Heat shock } 70 \mathrm{kDa} \\
\text { protein } 1 \mathrm{~A} / 1 \mathrm{~B}\end{array}$ & 0.86 & 4 & 0.86 & 7 & 0.19 & 7 & 0.25 & 8 \\
\hline HSPB1 & HSP beta-1 & -1.86 & 5 & -2.00 & 4 & 0.89 & 7 & 0.30 & 5 \\
\hline $\mathrm{K} 2 \mathrm{C} 8$ & $\begin{array}{l}\text { Keratin, type II } \\
\text { cytoskeletal } 8\end{array}$ & & & -2.36 & 8 & -0.92 & 9 & -0.90 & 4 \\
\hline NUCL & Nucleolin & -0.85 & 3 & -1.40 & 6 & -0.16 & 18 & 0.21 & 5 \\
\hline PRKDC & $\begin{array}{l}\text { DNA-dependent } \\
\text { protein kinase } \\
\text { catalytic subunit }\end{array}$ & & & -3.83 & 2 & -0.16 & 31 & 0.31 & 10 \\
\hline SERA & $\begin{array}{l}\text { D-3-Phosphoglycerate } \\
\text { dehydrogenase }\end{array}$ & -1.17 & 5 & -1.58 & 4 & -0.58 & 8 & -1.49 & 7 \\
\hline SYVC & Valyl-tRNA synthetase & & & -1.62 & 3 & -0.23 & 10 & -0.39 & 5 \\
\hline ТСРВ & $\begin{array}{l}\text { T-Complex protein } 1 \\
\text { subunit beta }\end{array}$ & & & -1.33 & 2 & 0.14 & 14 & -0.33 & 8 \\
\hline TKT & Transketolase & & & -1.45 & 5 & -0.36 & 14 & 0.20 & 2 \\
\hline $\mathrm{CH} 60$ & $\begin{array}{l}60 \text { kDa HSP, } \\
\text { mitochondrial }\end{array}$ & & & -0.90 & 5 & -0.25 & 28 & 0.25 & 6 \\
\hline ENPL & Endoplasmin & & & -0.82 & 4 & -0.18 & 26 & 0.20 & 13 \\
\hline EF2 & Elongation factor 2 & & & 0.73 & 3 & 0.21 & 26 & -0.25 & 9 \\
\hline
\end{tabular}


A

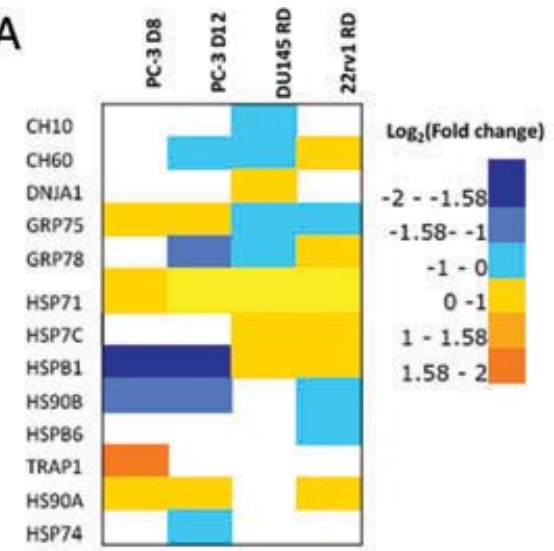

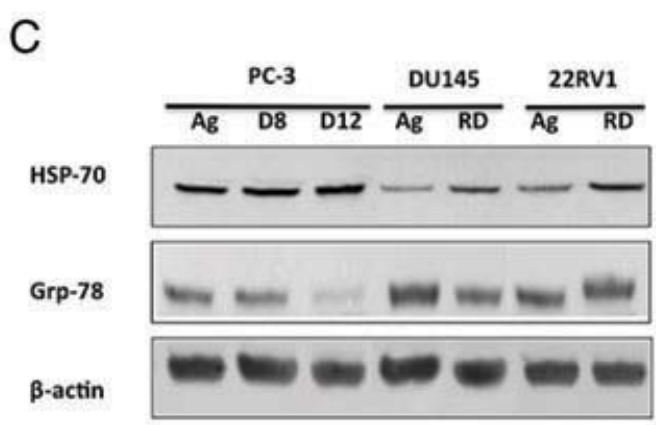

$E$
B

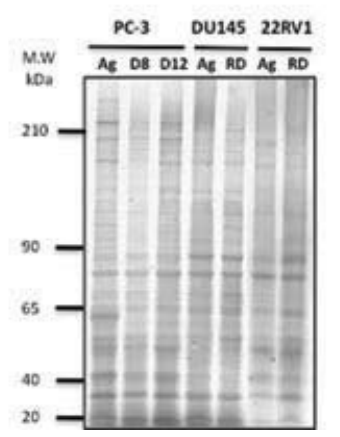

D

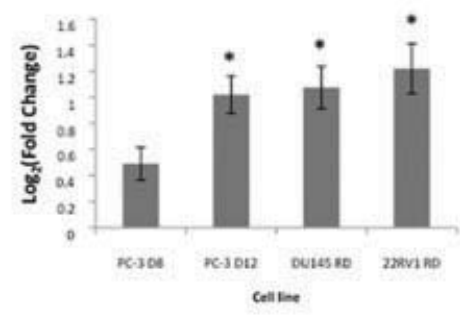

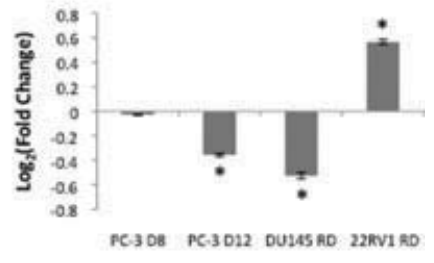

Cell Uine

Figure 2. Validation of altered chaperone protein abundance. (A) Heat map of fold changes in all chaperone proteins identified as having an altered abundance in docetaxel resistant cell lines, fold changes are calculated in $\log _{2}$ scale. Proteins are represented on the vertical axis while values for each individual resistant cell line are on the horizontal axis. (B) Representative image of $30 \mu \mathrm{g}$ of whole cell lysate, of the parental PC-3 cell line (Ag), both its docetaxel resistant variants (D8 and D12) and the parental cell lines DU145 (Ag) and $22 \mathrm{RV} 1$ (Ag) along with the corresponding docetaxel resistant variants (RD), separated on a 7.5\% 1D SDS-PAGE gel and stained with colloidal coomassie protein dye to demonstrate relative protein loading. (C) Western blot validation of HSP-70 (of which hsp71 and HSP7C are isoforms), also shown is the corresponding immunoblot for $\beta$-actin, which acted as a loading control; $2.5 \mu \mathrm{g}$ of whole cell lysate from each cell line was separated on a 7.5\% SDS-PAGE gel and subsequently transferred to nitrocellulose membrane. Graphical presentation of (D) HSP70 and (E) Grp78 fold changes in docetaxel-resistant cell lines. Values represent mean $\pm \operatorname{SD}(n=3)$, , a significant change with $p<0.05$ (Students $t$-test); fold change was calculated by comparing resistant variants to their respective parental cell line, following normalisation to $\beta$-actin based on densitometry measurements of band area, calculations carried out in $\log _{2}$ scale. Significant changes in HSP-70 abundance was detected in PC-3 D12, DU145 RD and 22RV1 RD docetaxel resistance cell lines, while Grp-78 demonstrated small but significant changes in abundance in the PC-3 D12 (decreased), DU145 RD (decreased) and 22RV1 RD (increased) docetaxel resistant cells.

\subsection{Validation of HSP alterations}

Widespread changes in HSP levels were identified in all docetaxel-resistant sub-lines (Fig. 2A). Of these, the HSP70 isoforms, HSP71 and HSP7C, had increased protein levels in two or more cell lines. Grp78, a regulator of endoplasmic reticulum homeostatsis, showed differential changes in expression across three resistant sub-lines. Validation of changes in the abundance of HSP70 and Grp78 was carried out by Western blot. Significant changes in HSP70 protein levels were confirmed in the PC-3 D12, DU145-RD and the 22RV1 sub-lines, with an increase of between 1.02 to $1.22 \log _{2}$ (fold change) in protein abundance across the four sub-lines (Fig. 2D and E). Grp78 had significant changes in all cell lines; PC-3 D12 (-0.35), DU145 RD (-0.556) and 22RV1 RD cells (0.56, Fig. 2D and E).

\subsection{Cytoskeletal changes}

A large number of cytoskeletal proteins were identified as having an altered abundance in docetaxel-resistant cells. A number of these changes are consistent with EMT, namely decreases in cytokeratin proteins and increases in vimentin and vinculin. It should be noted that keratin proteins are a common contaminant in MS experiments. Of the keratins identified in our study, see Table 2, K1C19 and cytokeratin 7 are known to be common contaminants [32]. However, all of the keratin proteins found to have altered abundance in the docetaxel resistant cell lines are expressed in normal prostatic tissue and prostate cancer cell lines ([33-35], EBI Gene Expression Atlas and Human Protein Atlas [36]). To fully confirm that these changes are not due to contamination, validation by another technique would be necessary. 
Table 2. Keratin proteins with an altered abundance in docetaxel resistant cell lines. Given are the number of unique peptides for quantification, a mascot score for protein identification, log ratio change in protein abundance and ANOVA $p$ values for the change. $p<$ 0.05 is deemed significant.

\begin{tabular}{|c|c|c|c|c|}
\hline Cell line & Peptides & Mascot score & ANOVA $(p)^{? ?}$ & $\log _{10}$ (fold change) \\
\hline K1C18_HUMAN & \multicolumn{4}{|c|}{ Keratin, type I cytoskeletal 18} \\
\hline PC-3 D8 & 5 & 616.92 & $1.42 \times 10^{-04}$ & -0.4456 \\
\hline PC-3 D12 & 6 & 631.45 & $9.19 \times 10^{-07}$ & -0.7275 \\
\hline DU145 RD & 8 & 907.49 & $2.45 \times 10^{-03}$ & -0.1038 \\
\hline 22RV1 RD & - & - & - & - \\
\hline K1C19_HUMAN & \multicolumn{4}{|c|}{ Keratin, type I cytoskeletal 19} \\
\hline PC-3 D8 & 2 & 202.52 & $4.46 \times 10^{-05}$ & -0.2989 \\
\hline PC-3 D12 & 6 & 487.95 & $4.13 \times 10^{-05}$ & -0.4166 \\
\hline DU145 RD & - & - & - & - \\
\hline 22RV1 RD & - & - & - & - \\
\hline K2C7_HUMAN & \multicolumn{4}{|c|}{ Keratin, type II cytoskeletal 7} \\
\hline PC-3 D8 & - & - & - & - \\
\hline PC-3 D12 & 5 & 323.09 & $1.55 \times 10^{-04}$ & -0.3181 \\
\hline DU145 RD & 2 & 1210.44 & 0.05 & -0.0334 \\
\hline 22RV1 RD & - & - & - & - \\
\hline K2C8_HUMAN & \multicolumn{4}{|c|}{ Keratin, type II cytoskeletal 8} \\
\hline PC-3 D8 & 3 & 203.1 & $1.20 \times 10^{-03}$ & -0.3385 \\
\hline PC-3 D12 & 8 & 510.32 & $2.93 \times 10^{-05}$ & -0.7118 \\
\hline DU145 RD & 8 & 796.43 & $1.42 \times 10^{-07}$ & -0.2765 \\
\hline 22RV1 RD & 4 & 209.35 & $6.97 \times 10^{-04}$ & -0.2695 \\
\hline KRT81_HUMAN & \multicolumn{4}{|c|}{ Keratin, type II cuticular Hb1 } \\
\hline PC-3 D8 & 3 & 208.77 & $2.15 \times 10^{-05}$ & -0.3979 \\
\hline PC-3 D12 & - & - & - & - \\
\hline DU145 RD & - & - & - & - \\
\hline 22RV1 RD & - & - & - & - \\
\hline
\end{tabular}

- No significant change in protein abundance detected.

So, our study validated two of these proteins by Western blotting using different protein lysates than employed in the LC-MS analysis. Consistent with the MS results, significant decreases in protein abundance for cytokeratin protein 18 (K1C18) were identified in PC-3 D12 and DU145 RD sublines. Similarly, cytokeratin 19 (K1C19) showed significant decreases in protein abundance in the PC-3 D12 sub-line (Fig. 3A). An additional decrease not identified by MS, in the abundance of K1C19, was found by Western blot in the DU145 RD sub-lines (Fig. 3A). Vinculin protein abundance was also assessed and showed a significant increase in the PC3 D12 sub-line (Fig. 3A). E-cadherin and N-cadherin are wellcharacterised markers of EMT, and additional Western blots were carried out to assess levels of these proteins in the docetaxel resistant cell lines. In both the PC-3 D12 and DU145 cell lines, E-cadherin levels were significantly decreased whereas $\mathrm{N}$-cadherin demonstrated increased expression in the PC-3 D8 and D12 cell lines. In the PC-3 D8 and D12 sub-lines, further indications of EMT were present. In Fig. 3(B), a change in morphology can be seen in the PC-3 D8 and D12 sublines when compared to the age-matched (Ag) parental cell line. However, no morphological changes were evident in the DU145 RD and 22RV1 RD sub-lines.

\subsection{AR abundance}

Pathway analysis showed that a number of the short-listed proteins had interactions with or regulated the expression of the AR. Hence, the expression of the AR in the docetaxelresistant sub-lines was investigated by Western blot. Interestingly, there was increased expression of the AR in the androgen-independent docetaxel-resistant cell line (significant in PC-3 D12 and the DU145 RD) as opposed to no change in protein abundance in the androgen-sensitive 22RV1 RD cell line (Fig. 4A and B [37]).

\section{Discussion}

To identify markers and to further understand the mechanisms of docetaxel resistance, four docetaxel-resistant cell lines were analysed by label-free LC-MS. Proteomic analysis revealed a small subset of proteins that consistently change with docetaxel resistance in a number of the cell lines. Validation of a subset of the identified proteins by Western blot confirmed the semi-quantitative findings of the LC-MS analysis. 

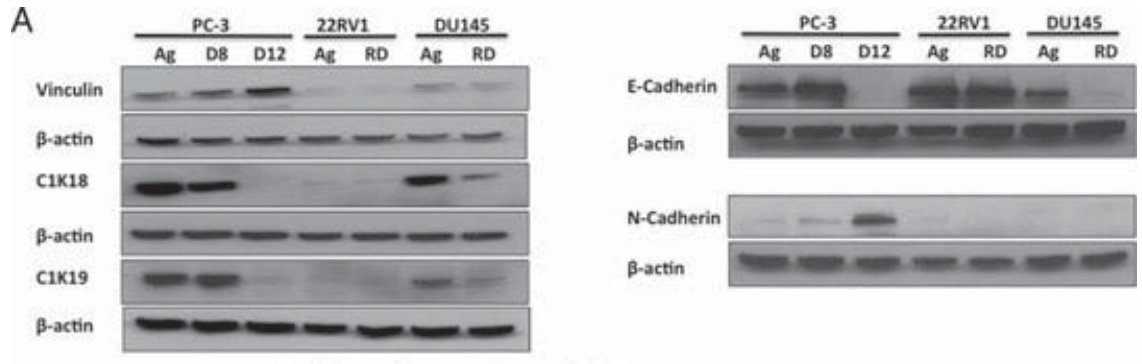

B

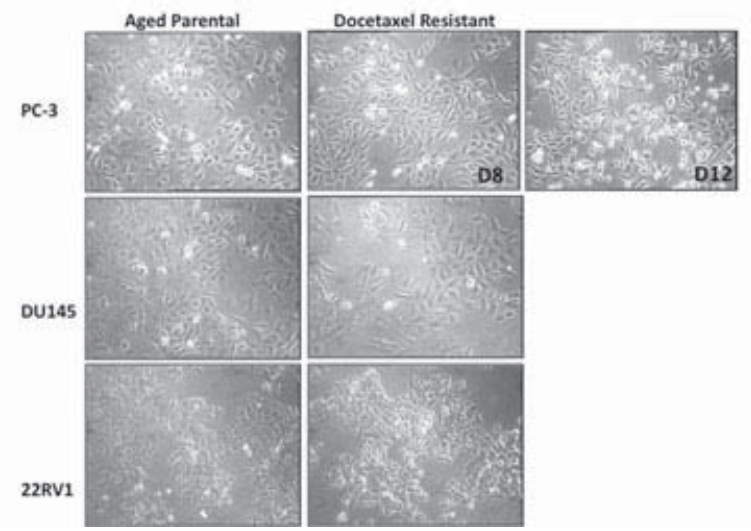

Figure 3. EMT association with docetaxel resistance. (A) Immunoblot comparison of known EMT markers; vinculin, cytokeratin 18 (C1K18), cytokeratin 19 (C1K19), ECadherin and $\mathrm{N}$-Cadherin, in the parental $\mathrm{PC}-3$ cell line $(\mathrm{Ag})$ and both its docetaxel resistant variants (D8 and D12) as well as the parental cell lines 22RV1 $(\mathrm{Ag})$ and DU145 (Ag) along with the corresponding docetaxel resistant variants (RD). A total of 30 $\mu \mathrm{g}$ of protein transferred to membranes, which were probed for known markers of EMT and then probed for $\beta$-actin, which acted as a loading control. Vinculin has increased abundance in the PC-3 D12 cell line. C1K18 and C1K19 both demonstrate decreased abundance in the PC-3 D12 and DU145 docetaxel resistant cell lines. E-Cadherin abundance decreased significantly in both the PC-3 D12 and the DU145 cell lines. While $\mathrm{N}$-cadherin was only detectable in the PC-3 cell lines and displayed increased expression in the PC-3 D8 and D12 docetaxel resistant variants. (B) Cell images of parental and resistant variants of PC-3, DU145 and 22RV1 cell lines (20× magnification). Morphological changes can be seen in PC-3 D8 and D12 docetaxel resistant cells.
A

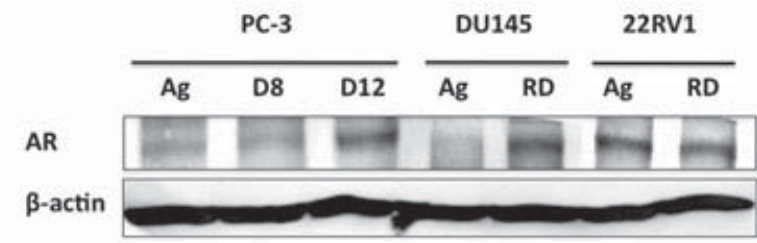

B

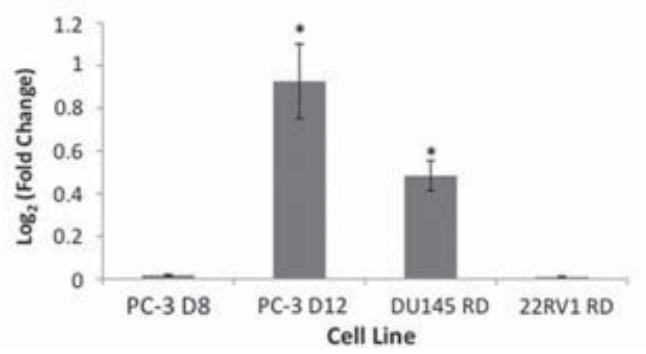

Figure 4. Altered abundance of the androgen receptor (AR). (A) Immunoblot analysis of the AR; in the parental PC-3 cell line $(\mathrm{Ag})$, both its docetaxel resistant variants (D8 and D12) and in the parental cell lines DU145 (Ag) and 22RV1 (Ag) along with the corresponding docetaxel resistant variants (RD). Also shown is the corresponding immunoblot for $\beta$-actin, which acted as a loading control. (B) Graphical presentation of AR $\log _{2}$ (fold change) in docetaxel resistant cell lines. Values represent mean \pm SD $(n=3),{ }^{*}$ a significant change with $p<0.05$ (Students $t$-test) fold change was calculated by comparing band area (normalised to $\beta$-actin) of resistant variants to their respective parental cell line.
The approach taken by this study has identified four proteins that show concurrent changes in protein abundance in the docetaxel resistant cell lines examined. K2C8, a cytokeratin, was decreased in docetaxel resistant cells, HSP70, a HSP, had increased abundance in docetaxel resistant cells; both will be discussed in more detail with respect to cellular processes. D-3-phosphoglycerate dehydrogenase (SERA) catalyses the rate-limiting step of serine biosynthesis, 3-phosphoglycerate into 3-phosphohydroxypyruvate. It is present in higher levels in approximately $70 \%$ of estrogen receptor negative breast cancers but has not been linked to prostate cancer or docetaxel resistance previously [38]. Valyl-tRNA synthetase (SYVC) catalyses the attachment of amino acids to their complementary tRNA. The changes to K2C8 and SERA concur with the findings of Zhao et al., [28], while the changes to HSP70 and SYVC have not previously been associated with docetaxel resistance in prostate cancer.

\subsection{Epithelial-to-mesenchymal transition}

EMT, the transition of cells from an epithelial to mesenchymal phenotype, occurs during cancer progression and can contribute to the migration and metastasis of cancer cells [39-41]. EMT has been associated with docetaxel resistance in breast cancer [42] and in resistance to other chemotherapeutics $[43,44]$. In this study, docetaxel-resistant sub-lines exhibited widespread changes in protein markers of EMT when analysed by LC-MS. Further analysis of EMT-associated 
markers by Western blot suggests the occurrence of EMT in both the PC-3 D12 and the DU145 RD cell lines. This is supported by morphological changes evident in the PC-3 docetaxel resistant cell lines. Previous studies, such as those by Zhao et al., [28] have also examined proteomic changes due to docetaxel resistance in PC_3 resistant variants that have a high level of resistance to docetaxel without any apparent change in cellular morphology. Previously, it has been shown that treating these PC-3 D12 and DU145 RD cells with docetaxel induces NFкB activation [29]. It has been suggested that NFKB activation is key to EMT [45], therefore induced alterations of NFKB activity may suggest a mechanism for the EMT induction in these cells. Further functional work will be necessary to determine if EMT contributes to resistance or is merely a manifestation of docetaxel-induced changes in cytoskeletal proteins.

\subsection{HSPs}

HSPs act as molecular chaperones, aiding the folding of proteins under normal conditions and play a key role in cellular response to stress [46,47]. Since HSPs have such a vital role in protecting cells from damage and have such a wide range of client proteins, they have become exciting drug targets in the treatment of cancer [48-50]. Proteomic analysis of the three docetaxel resistant sub-lines revealed increases in proteins such as HSP90a and HSP70 in multiple cell lines. HSP90 is frequently over-expressed in cancer cells, playing a protective role by repairing damage induced by cytotoxic chemotherapies and preventing the degradation of mutated oncoproteins [51]. HSP90 client proteins include a wide range of proteins key to prostate cancer development and progression, such the AR. Targeting HSP90 function with specific inhibitors affects the stability and function of these proteins, as such a number of HSP90 inhibitors are under development or in clinical trails [52]. 17-AAG, a geldanamycin derivative, has an effect on AR receptor status in vivo and has been shown to delay the progression to castrate resistance in xenograft models of prostate cancer [53,54]. Grp78, a member of the HSP70 family, is induced as a result of endoplasmic reticulum stress. It is associated with the development of advanced metastatic prostate cancer and poorer survival $[55,56]$. Grp78 expression decreased in the androgen-independent PC-3 D12 and DU145 RD sub-lines but increased in the androgensensitive 22RV1 RD line. The differential expression of Grp78 highlights the importance of examining proteins in multiple cell lines. HSP70 also known as HSP71 and HSPA1A is at present in low levels in normal cells. It is rapidly induced under conditions of cellular stress and prevents stressinduced apoptosis $[57,58]$. HSP70 (HSP71) is upregulated in several docetaxel resistant cell lines. It and has previously been implicated in resistance to cisplatin resistance and other chemotherapeutic agents $[59,60]$. Targeting HSP70 expression in animal and cell models of cancer has anti-tumour effects [61, 62]. HSP70 abundance is increased in docetaxel resistant cells. However, targeting a single HSP in isolation may not be an effective treatment strategy, as many HSPs have overlapping effects and associated functions. For example, AAG-17 treatment induces HSP70 expression, which may limit the effectiveness of this HSP90 inhibitor [63], Targeting multiple HSPs in combination with existing therapies may be a more effective treatment strategy that could be applied to docetaxel resistant CRPC, and highlights the need for further development of HSP inhibitor strategies and pharmaceutical.

\subsection{Docetaxel-resistant CRPC cells express higher levels of the AR}

The AR is expressed throughout prostate cancer progression [64] and is expressed in the PC-3 and DU145 cell lines despite the general belief that these cell lines are AR negative [65]. Protein expression changes in docetaxel resistant cells, identified by label-free LC-MS, were linked to AR regulation and expression by additional bioinformatic analysis [66-69]. In this study, all cell lines, parental and docetaxel resistant, expressed AR. In the androgen-independent docetaxel resistant cell lines, PC-3 D12 and DU145 RD, AR expression was increased when compared to parental cells. In docetaxel sensitive cells, AR signalling is impaired by docetaxel treatment, as nuclear localisation of the receptor is inhibited [70]. Further work will determine if this inhibition of AR signalling is present in docetaxel resistant cells, and contributes to their growth, as it has been shown that in some CPRC cell lines AR expression can affect growth [71].

Quantitative label-free LC-MS analysis may represent an attractive tool for predictive biomarker discovery and/or aid in the mechanistic understanding of phenotypic alterations in cells, e.g. those associated with resistance, since it can be used both to identify unknown proteins and provide comparative quantitative information on their abundance. However, proteomic analysis is limited to a subset of proteins that can be identified and quantified. This study utilised complementary bioinformatic techniques to get a broader perspective on the individual protein changes generated from the proteomic analysis and thereby identify linkages and pathways that might act as rational targets for evaluation. These pathway and system targets may not have been identified using alternative techniques that do not examine the interrelationships of the findings. Based on the proteomic profiling of docetaxel resistant prostate cancer cells, EMT may have an association with docetaxel resistance in prostate cancer. HSPs have emerged as potential drug targets that warrant further investigation in docetaxel resistant prostate cancer and our analysis suggests that the development of docetaxel resistance may be associated with increased AR expression in at least some instances. Furthermore, this analysis has identified protein changes not limited to a single cell line, which may act as more robust markers of docetaxel resistance. These include SERA, SYVC, HSP70 and K2C8, which have altered 
expression in all the docetaxel resistant cell lines examined. Further analysis will indicate if these proteins represent useful biomarkers of resistance and/or targets for drug intervention, which aid in improving docetaxel-based treatment efficacy.

The authors wish to thank the Science Foundation Ireland, Strategic Research Cluster award to Molecular Therapeutics for Cancer Ireland (award 08/SRC/B1410) for funding this work. SFI PI grant (03/IN3/B395) and SFI Equipment Grant (03/IN3B395/EC07) and PRTLI cycle III for funding the purchase of equipment used in this study.

The authors have declared no conflict of interest.

\section{References}

[1] Jemal, A., Siegel, R., Xu, J., Ward, E., Cancer statistics, 2010. CA Cancer J. Clin. 2011, 61, 133-134.

[2] Siegel, R., Ward, E., Brawley, O., Jemal, A., Cancer statistics, 2011: the impact of eliminating socioeconomic and racial disparities on premature cancer deaths. CA Cancer J. Clin. 2011, 61, 212-236.

[3] Hayat, M. J., Howlader, N., Reichman, M. E., Edwards, B. K., Cancer statistics, trends, and multiple primary cancer analyses from the Surveillance, Epidemiology, and End Results (SEER) Program. Oncologist 2007, 12, 20-37.

[4] Denmeade, S. R., Lin, X. S., Isaacs, J. T., Role of programmed (apoptotic) cell death during the progression and therapy for prostate cancer. Prostate 1996, 28, 251-265.

[5] Hobisch, A., Culig, Z., Radmayr, C., Bartsch, G. et al., Distant metastases from prostatic carcinoma express androgen receptor protein. Cancer Res. 1995, 55, 30683072.

[6] Tannock, I. F., de Wit, R., Berry, W. R., Horti, J. et al., Docetaxel plus prednisone or mitoxantrone plus prednisone for advanced prostate cancer. N. Engl. J. Med. 2004, 351, 15021512.

[7] Petrylak, D. P., Tangen, C. M., Hussain, M. H., Lara, P. N., Jr. et al., Docetaxel and estramustine compared with mitoxantrone and prednisone for advanced refractory prostate cancer. N. Engl. J. Med. 2004, 351, 1513-1520.

[8] Urakami, S., Igawa, M., Kikuno, N., Yoshino, T. et al., Combination chemotherapy with paclitaxel, estramustine and carboplatin for hormone refractory prostate cancer. J. Urol. 2002, 168, 2444-2450.

[9] Berthold, D. R., Pond, G. R., Soban, F., de Wit, R. et al., Docetaxel plus prednisone or mitoxantrone plus prednisone for advanced prostate cancer: updated survival in the TAX 327 study. J. Clin. Oncol. 2008, 26, 242-245.

[10] Baker, S. D., Sparreboom, A., Verweij, J., Clinical pharmacokinetics of docetaxel: recent developments. Clin. Pharmacokinet 2006, 45, 235-252.

[11] Bruno, R., Hille, D., Riva, A., Vivier, N. et al., Population pharmacokinetics/pharmacodynamics of docetaxel in phase
II studies in patients with cancer. J. Clin. Oncol. 1998, 16, 187-196.

[12] Di Lorenzo, G., Buonerba, C., Faiella, A., Rescigno, P. et al., Phase II study of docetaxel re-treatment in docetaxelpretreated castration-resistant prostate cancer. BJU Int. 2011, 107, 234-239.

[13] Raghavan, D., Koczwara, B., Javle, M., Evolving strategies of cytotoxic chemotherapy for advanced prostate cancer. Eur. J. Cancer 1997, 33, 566-574.

[14] Ploussard, G., Terry, S., Maille, P., Allory, Y. et al., Class III beta-tubulin expression predicts prostate tumor aggressiveness and patient response to docetaxel-based chemotherapy. Cancer Res. 70, 9253-9264.

[15] McMenamin, M. E., Soung, P., Perera, S., Kaplan, I. et al., Loss of PTEN expression in paraffin-embedded primary prostate cancer correlates with high Gleason score and advanced stage. Cancer Res. 1999, 59, 4291-4296.

[16] Feldman, B. J., Feldman, D., The development of androgenindependent prostate cancer. Nat. Rev. Cancer 2001, 1, 34 45.

[17] McEleny, K., Coffey, R., Morrissey, C., Williamson, K. et al., An antisense oligonucleotide to clAP-1 sensitizes prostate cancer cells to fas and TNFalpha mediated apoptosis. Prostate 2004, 59, 419-425.

[18] Patterson, S. G., Wei, S., Chen, X., Sallman, D. A. et al., Novel role of Stat1 in the development of docetaxel resistance in prostate tumor cells. Oncogene 2006, 25, 61136122.

[19] Zhong, B., Sallman, D. A., Gilvary, D. L., Pernazza, D. et al., Induction of clusterin by AKT-role in cytoprotection against docetaxel in prostate tumor cells. Mol. Cancer Ther. 9, 18311841.

[20] Gibbons, N. B., Watson, R. W., Coffey, R. N., Brady, H. P. et al., Heat-shock proteins inhibit induction of prostate cancer cell apoptosis. Prostate 2000, 45, 58-65.

[21] Li, X. H., Li, C., Xiao, Z. Q., Proteomics for identifying mechanisms and biomarkers of drug resistance in cancer. J. Proteomics 74, 2642-2649.

[22] Fredolini, C., Liotta, L. A., Petricoin, E. F., Application of proteomic technologies for prostate cancer detection, prognosis, and tailored therapy. Crit. Rev. Clin. Lab Sci. 2010, 47, 125-138.

[23] Catalona, W. J., Smith, D. S., Ratliff, T. L., Dodds, K. M. et al., Measurement of prostate-specific antigen in serum as a screening test for prostate cancer. N. Engl. J. Med. 1991, $324,1156-1161$.

[24] Aebersold, R., Mann, M., Mass spectrometry-based proteomics. Nature 2003, 422, 198-207.

[25] Sardana, G., Jung, K., Stephan, C., Diamandis, E. P., Proteomic analysis of conditioned media from the PC3, LNCaP, and 22Rv1 prostate cancer cell lines: discovery and validation of candidate prostate cancer biomarkers. J. Proteome Res. 2008, 7, 3329-3338.

[26] M'Koma, A. E., Blum, D. L., Norris, J. L., Koyama, T. et al., Detection of pre-neoplastic and neoplastic prostate disease by MALDI profiling of urine. Biochem. Biophys. Res. Commun. 2007, 353, 829-834. 
[27] Glen, A., Gan, C. S., Hamdy, F. C., Eaton, C. L. et al., iTRAQfacilitated proteomic analysis of human prostate cancer cells identifies proteins associated with progression. J. Proteome Res. 2008, 7, 897-907.

[28] Zhao, L., Lee, B. Y., Brown, D. A., Molloy, M. P. et al., Identification of candidate biomarkers of therapeutic response to docetaxel by proteomic profiling. Cancer Res. 2009, 69, 7696-7703.

[29] O’Neill, A. J., Prencipe, M., Dowling, C., Fan, Y. et al., Characterisation and manipulation of docetaxel resistant prostate cancer cell lines. Mol. Cancer 10, 126. doi: 10.1186/14764598-10-126

[30] Duan, X., Young, R., Straubinger, R. M., Page, B. et al., A straightforward and highly efficient precipitation/on-pellet digestion procedure coupled with a long gradient nano-LC separation and Orbitrap mass spectrometry for label-free expression profiling of the swine heart mitochondrial proteome. J. Proteome Res. 2009, 8, 2838-2850.

[31] Nikitin, A., Egorov, S., Daraselia, N., Mazo, I., Pathway studio-the analysis and navigation of molecular networks. Bioinformatics 2003, 19, 2155-2157.

[32] Keller, B. O., Sui, J., Young, A. B., Whittal, R. M., Interferences and contaminants encountered in modern mass spectrometry. Anal. Chim. Acta 2008, 627, 71-81.

[33] van Bokhoven, A., Varella-Garcia, M., Korch, C., Johannes, W. U. et al., Molecular characterization of human prostate carcinoma cell lines. Prostate 2003, 57, 205-225.

[34] Dozmorov, M. G., Hurst, R. E., Culkin, D. J., Kropp, B. P. et al., Unique patterns of molecular profiling between human prostate cancer LNCaP and PC-3 cells. Prostate 2009, 69, 1077-1090.

[35] Uchida, K., Chaudhary, L. R., Sugimura, Y., Adkisson, H. D. et al., Proprotein convertases regulate activity of prostate epithelial cell differentiation markers and are modulated in human prostate cancer cells. J. Cell Biochem. 2003, 88, 394399.

[36] Uhlen, M., Oksvold, P., Fagerberg, L., Lundberg, E. et al., Towards a knowledge-based human protein atlas. Nat. Biotechnol. 28, 1248-1250.

[37] Tepper, C. G., Boucher, D. L., Ryan, P. E., Ma, A. H. et al., Characterization of a novel androgen receptor mutation in a relapsed CWR22 prostate cancer xenograft and cell line. Cancer Res. 2002, 62, 6606-6614.

[38] Possemato, R., Marks, K. M., Shaul, Y. D., Pacold, M. E. et al., Functional genomics reveal that the serine synthesis pathway is essential in breast cancer. Nature 476, 346-350.

[39] Thiery, J. P., Epithelial-mesenchymal transitions in tumour progression. Nat. Rev. Cancer 2002, 2, 442-454.

[40] Kiemer, A. K., Takeuchi, K., Quinlan, M. P., Identification of genes involved in epithelial-mesenchymal transition and tumor progression. Oncogene 2001, 20, 6679-6688.

[41] Savagner, P., The epithelial-mesenchymal transition (EMT) phenomenon. Ann. Oncol. 2010, 21(Suppl 7), vii89-vii92.

[42] Iseri, O. D., Kars, M. D., Gunduz, U., Two different docetaxel resistant MCF-7 sublines exhibited different gene expression pattern. Mol. Biol. Rep. 2011, 39, 3505-3516.

[43] Kajiyama, H., Shibata, K., Terauchi, M., Yamashita, M. et al., Chemoresistance to paclitaxel induces epithelialmesenchymal transition and enhances metastatic potential for epithelial ovarian carcinoma cells. Int. J. Oncol. 2007, 31, 277-283.

[44] Hiscox, S., Jiang, W. G., Obermeier, K., Taylor, K. et al., Tamoxifen resistance in MCF7 cells promotes EMT-like behaviour and involves modulation of beta-catenin phosphorylation. Int. J. Cancer 2006, 118, 290-301.

[45] Huber, M. A., Azoitei, N., Baumann, B., Grunert, S. et al., NF-kappaB is essential for epithelial-mesenchymal transition and metastasis in a model of breast cancer progression. J. Clin. Invest 2004, 114, 569-581.

[46] Lindquist, S., Craig, E. A., The heat-shock proteins. Annu. Rev. Genet 1988, 22, 631-677.

[47] Kampinga, H. H., Chaperones in preventing protein denaturation in living cells and protecting against cellular stress. Handb. Exp. Pharmacol. 2006, 172, 1-42.

[48] Bagatell, R., Whitesell, L., Altered Hsp90 function in cancer: a unique therapeutic opportunity. Mol. Cancer Ther. 2004, 3, 1021-1030.

[49] Isaacs, J. S., Xu, W., Neckers, L., Heat shock protein 90 as a molecular target for cancer therapeutics. Cancer Cell 2003, 3, 213-217.

[50] Kim, Y., Lillo, A. M., Steiniger, S. C., Liu, Y. et al., Targeting heat shock proteins on cancer cells: selection, characterization, and cell-penetrating properties of a peptidic GRP78 ligand. Biochemistry 2006, 45, 9434-9444.

[51] Whitesell, L., Lindquist, S. L., HSP90 and the chaperoning of cancer. Nat. Rev. Cancer 2005, 5, 761-772.

[52] Neckers, L., Hsp90 inhibitors as novel cancer chemotherapeutic agents. Trends Mol. Med. 2002, 8, S55-S61.

[53] Solit, D. B., Zheng, F. F., Drobnjak, M., Munster, P. N. et al., 17Allylamino-17-demethoxygeldanamycin induces the degradation of androgen receptor and HER-2/neu and inhibits the growth of prostate cancer xenografts. Clin. Cancer Res. 2002, 8, 986-993.

[54] O'Malley, K. J., Langmann, G., Ai, J., Ramos-Garcia, R. et al., Hsp90 inhibitor 17-AAG inhibits progression of LuCaP35 xenograft prostate tumors to castration resistance. Prostate 2011. doi: 10.1002/pros.22458

[55] Pootrakul, L., Datar, R. H., Shi, S. R., Cai, J. et al., Expression of stress response protein Grp78 is associated with the development of castration-resistant prostate cancer. Clin. Cancer Res. 2006, 12, 5987-5993.

[56] Daneshmand, S., Quek, M. L., Lin, E., Lee, C. et al., Glucoseregulated protein GRP78 is up-regulated in prostate cancer and correlates with recurrence and survival. Hum. Pathol. 2007, 38, 1547-1552.

[57] Tan, S. S., Ahmad, I., Bennett, H. L., Singh, L. et al., GRP78 up-regulation is associated with androgen receptor status, Hsp70-Hsp90 client proteins and castrate-resistant prostate cancer. J. Pathol. 223, 81-87.

[58] Ravagnan, L., Gurbuxani, S., Susin, S. A., Maisse, C. et al., Heat-shock protein 70 antagonizes apoptosis-inducing factor. Nat. Cell Biol. 2001, 3, 839-843.

[59] Gabai, V. L., Budagova, K. R., Sherman, M. Y., Increased expression of the major heat shock protein Hsp72 in human 
prostate carcinoma cells is dispensable for their viability but confers resistance to a variety of anticancer agents. Oncogene 2005, 24, 3328-3338.

[60] Ren, A., Yan, G., You, B., Sun, J., Down-regulation of mammalian sterile 20 -like kinase 1 by heat shock protein 70 mediates cisplatin resistance in prostate cancer cells. Cancer Res. 2008, 68, 2266-2274.

[61] Schmitt, E., Maingret, L., Puig, P. E., Rerole, A. L. et al., Heat shock protein 70 neutralization exerts potent antitumor effects in animal models of colon cancer and melanoma. Cancer Res. 2006, 66, 4191-4197.

[62] Zhao, Z. G., Shen, W. L., Heat shock protein 70 antisense oligonucleotide inhibits cell growth and induces apoptosis in human gastric cancer cell line SGC-7901. World J. Gastroenterol. 2005, 11, 73-78.

[63] Goetz, M. P., Toft, D., Reid, J., Ames, M. et al., Phase I trial of 17-allylamino-17-demethoxygeldanamycin in patients with advanced cancer. J. Clin. Oncol. 2005, 23, 1078-1087.

[64] van der Kwast, T. H., Schalken, J., Ruizeveld de Winter, J. A., van Vroonhoven, C. C. et al., Androgen receptors in endocrine-therapy-resistant human prostate cancer. Int. J. Cancer 1991, 48, 189-193.

[65] Alimirah, F., Chen, J., Basrawala, Z., Xin, H. et al., DU-145 and PC-3 human prostate cancer cell lines express androgen receptor: implications for the androgen receptor functions and regulation. FEBS Lett. 2006, 580, 2294-2300.

[66] Frigo, D. E., McDonnell, D. P., Differential effects of prostate cancer therapeutics on neuroendocrine transdifferentiation. Mol. Cancer Ther. 2008, 7, 659-669.

[67] Veldscholte, J., Berrevoets, C. A., Brinkmann, A. O., Grootegoed, J. A. et al., Anti-androgens and the mutated androgen receptor of LNCaP cells: differential effects on binding affinity, heat-shock protein interaction, and transcription activation. Biochemistry 1992, 31, 2393-2399.

[68] Loy, C. J., Sim, K. S., Yong, E. L., Filamin-A fragment localizes to the nucleus to regulate androgen receptor and coactivator functions. Proc. Natl. Acad. Sci. USA 2003, 100, 4562-4567.

[69] Leav, I., Plescia, J., Goel, H. L., Li, J. et al., Cytoprotective mitochondrial chaperone TRAP-1 as a novel molecular target in localized and metastatic prostate cancer. Am. J. Pathol. $176,393-401$.

[70] Zhu, M. L., Horbinski, C. M., Garzotto, M., Qian, D. Z. et al., Tubulin-targeting chemotherapy impairs androgen receptor activity in prostate cancer. Cancer Res. 70, 7992-8002.

[71] Compagno, D., Merle, C., Morin, A., Gilbert, C. et al., SIRNAdirected in vivo silencing of androgen receptor inhibits the growth of castration-resistant prostate carcinomas. PLoS One 2007, 2, e1006. 